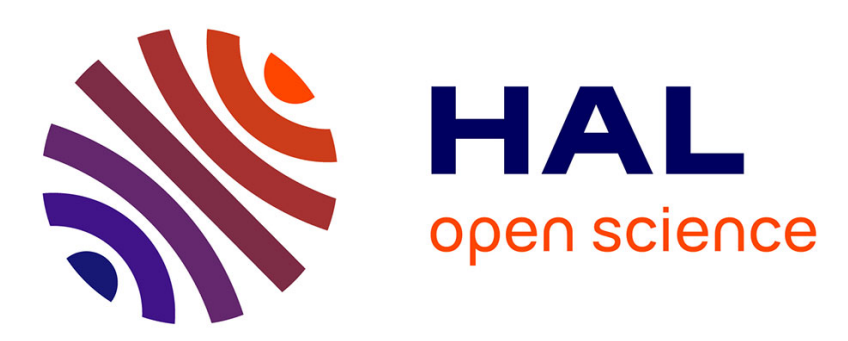

\title{
Synchronized B and 13C Diamond Delta Structures for an Ultimate In-Depth Chemical Characterization
}

Alexandre Fiori, François Jomard, Tokuyuki Teraji, Satoshi Koizumi, Junichi Isoya, Etienne Gheeraert, Etienne Bustarret

\section{- To cite this version:}

Alexandre Fiori, François Jomard, Tokuyuki Teraji, Satoshi Koizumi, Junichi Isoya, et al.. Synchronized B and 13C Diamond Delta Structures for an Ultimate In-Depth Chemical Characterization. Japanese Journal of Applied Physics, part 2: Letters, 2013, 6, pp.045801. 10.7567/APEX.6.045801 . hal-00838943

\section{HAL Id: hal-00838943 \\ https://hal.science/hal-00838943}

Submitted on 26 Jun 2013

HAL is a multi-disciplinary open access archive for the deposit and dissemination of scientific research documents, whether they are published or not. The documents may come from teaching and research institutions in France or abroad, or from public or private research centers.
L'archive ouverte pluridisciplinaire HAL, est destinée au dépôt et à la diffusion de documents scientifiques de niveau recherche, publiés ou non, émanant des établissements d'enseignement et de recherche français ou étrangers, des laboratoires publics ou privés. 


\title{
Title
}

Synchronized B and ${ }^{13} \mathrm{C}$ Diamond Delta Structures for an Ultimate In-Depth Chemical Characterization Authors

Alexandre Fiori ${ }^{*}{ }^{1,2}$, François Jomard ${ }^{3}$, Tokuyuki Teraji ${ }^{2}$, Satoshi Koizumi ${ }^{2}$, Junichi Isoya ${ }^{4}$, Etienne Gheeraert $^{1}$, and Etienne Bustarret ${ }^{1}$

\section{Affiliations}

${ }^{1}$ Institut Néel, CNRS and Université Joseph Fourier, BP 166, 38042 Grenoble Cedex 9, France

${ }^{2}$ National Institute for Materials Science, 1-1 Namiki, Tsukuba, Ibaraki, 305-0044, Japan

${ }^{3} \mathrm{GEMaC}, \mathrm{CNRS}$ and Université Versailles St Quentin, 45, avenue des Etats-Unis, 78035 Versailles

Cedex, France

${ }^{4}$ Tsukuba University, 1-2 Kasuga, Tsukuba, Ibaraki 305-8550, Japan

*E-mail address: FIORI.Alexandre@ @ims.go.jp

\begin{abstract}
The nanometer-range depth resolution of secondary ion mass spectrometry (SIMS) profiles in diamond was achieved by the determination of the depth resolution function (DRF). The measurement of this DRF was performed thanks to isotopic-enriched diamond delta structures composed of ${ }^{12} \mathrm{C}$ and ${ }^{13} \mathrm{C}$. The artificial SIMS broadening observed on the ${ }^{13} \mathrm{C}$ depth profiles of buried doped diamond epilayers was eliminated and replaced by a boxlike ${ }^{13} \mathrm{C}$ depth profile. Applied to boron delta-doped diamond structures, this analysis has resolved edge widths close to $0.3 \mathrm{~nm} / \mathrm{dec}$, as compared with $1.5 \mathrm{~nm} / \mathrm{decade}$ on the raw SIMS data.
\end{abstract}


Diamond has several excellent characteristics, superior to those of other semiconductors, e.g., Si

and $\mathrm{SiC}$. The high breakdown voltage and high temperature operation of diamond have strongly motivated research aiming at the development of next-generation high-power devices. ${ }^{1)}$ More in line with the optical properties that have given diamond its value as a jewel, modern diamond research is also focused on photonics and spintronics related to colour centers, e.g., NV centers in diamond. ${ }^{2-3)}$ Both types of application require the availability of very thin doped layers (boron or nitrogen-doped) in the range of nanometer thickness, the so-called "delta structures", 3-5) as well as the possibility to characterize such ultrathin epilayers.

Secondary ion mass spectrometry (SIMS) is commonly used to obtain depth profiles of dopant concentrations in diamond and other semiconductors. This technique uses a bombardment by energetic ions (usually $\mathrm{Cs}^{+}$or $\mathrm{O}_{2}^{+}$) to sputter and simultaneously mass-select recoiled secondary ions. The depth resolution of the depth concentration profile is mainly limited by primary ion masses, by incident and detected ion kinetic energies, by the incidence and collection angles, and by the roughness of the sample. Indeed, a SIMS-induced ion mixing is particularly visible when two adjacent layers, in the same sample, have a large and abrupt difference in doping level or in chemical composition. Below 100 $\mathrm{nm}$ in thickness, this effect is no longer negligible; it strongly affects the depth profile measurements by broadening, so that the raw SIMS profile $\left(\mathrm{P}_{\text {SIMS }}\right)$ differs from the dopant profile $\left(\mathrm{P}_{\text {Dopant }}\right)$, up to the point where the thickness values and atom peak concentrations in multilayer stacks become erroneous.

Technically, during a SIMS analysis, the experimental depth profile $\left(\mathrm{P}_{\text {SIMS }}\right)$ is the convolution of the dopant depth profile $\left(\mathrm{P}_{\text {Dopant }}\right)$ and of the depth resolution function $(\mathrm{DRF}) .{ }^{6)}$ Evaluation of this DRF (which depends on the probed atom) is a key issue in nanometer-range secondary ion mass spectrometry and a crucial step toward the prediction of artefacts affecting measured SIMS depth profiles. Deconvolution analysis using such a DRF provides accurate measurements on steep dopant depth profiles over many orders of magnitude in concentration. In the context of delta doping, the large dynamical doping range is a key advantage of SIMS when compared with other nanometric chemical 
1 profiling methods such as elastic recoil detection (ERD) ${ }^{7)}$, atom probe tomography ${ }^{8)}$ or transmission

2 electron microcopy-related techniques, ${ }^{9,10)}$ or to more "indirect" probes such as magneto-transport 3 measurements. $^{11)}$

Previous SIMS data processing schemes, especially in the case of diamond boron doping, have

5 been based on 4-points smoothing techniques, ${ }^{4)}$ but do not deal with ion mixing directly, although this

6 physical process is known to be responsible of the specific exponential shape of the trailing edges in

7 impurity profiles where a sharp drop of the impurity concentration with depth is expected. To estimate

8 quantitatively the influence of ion mixing occurring in the diamond during the SIMS analysis (for a

9 given crystalline orientation and surface roughness), local isotopic substitution (or "isotopically pure

10 growth") must be employed, similar to the case of silicon $\left({ }^{28} \mathrm{Si} /{ }^{30} \mathrm{Si}\right) .{ }^{12)}$ Isotope substitution is the ideal

control experiment to extract the best DRF fit, because it introduces only a negligible difference in mass (same recoiling effect) and no additional crystalline strain (same lattice parameter). In diamond, carbon is found with two stable isotopes: ${ }^{12} \mathrm{C}$ and ${ }^{13} \mathrm{C}$, and isotopically modulated epitaxial multilayers 
layer requires strict conditions such as a flat surface, no chemical diffusion in the matter, and a crystalline substrate. ${ }^{14)}$ Diamond epitaxial multilayer stacks fulfill these requirements.

According to experiments in silicon, ${ }^{15)}$ the SIMS depth profile corresponding to an isotopic deltadoped layer is not a sharp rectangular-shaped profile, but an arrangement of a Gaussian-like rounded top with exponentials rising and trailing edges. In this study, we use an analytical expression of the DRF proposed by Dowsett et al., ${ }^{14)}$ which results in the convolution of a double exponential, governed by $\lambda_{\text {up }}$ and $\lambda_{\text {down }}$ parameters, with a Gaussian function, where $\sigma$ is related to the full width at half maximum, as follows:

$\operatorname{DRF}(z)=\frac{1}{2\left(\lambda_{\text {up }}+\lambda_{\text {down }}\right)} \times\left\{\exp \left(\frac{z-z_{0}}{\lambda_{\text {up }}}+\frac{\sigma^{2}}{2 \lambda_{\text {up }}^{2}}\right) \times\left[1+\frac{1}{\sqrt{2}} \cdot \operatorname{erf}\left(\frac{-\left(z-z_{0}\right)}{\sigma}-\frac{\sigma}{\lambda_{\text {up }}}\right)\right]+\exp \left(\frac{-\left(z-z_{0}\right)}{\lambda_{\text {down }}}+\right.\right.$ $\left.\left.\frac{\sigma^{2}}{2 \lambda_{\text {down }}^{2}}\right) \times\left[1+\frac{1}{\sqrt{2}} \cdot \operatorname{erf}\left(\frac{z-z_{0}}{\sigma}-\frac{\sigma}{\lambda_{\text {down }}}\right)\right]\right\},(1)$.

A fourth parameter $z_{0}$ represents the position of the cusp of the double exponential.

Mathematically, the DRF can be plotted as a depth profile after being convoluted with a Dirac delta function localized at $z_{0}$, which has no thickness and a maximal top value [see Fig. 1(a)]. SIMS analysis is usually performed from the sample surface to the substrate, and the ion mixing (forward scattering of atoms) is then effective from the left to the right on the plot, explaining the profile asymmetry visible in the Figs. $1-3$ as well as in our previous measurements. ${ }^{5)}$ Consequently, the "rising" interface (located above the delta layer) will always show a sharper edge on the original SIMS profile compared with the broad "falling" tail located below the layer $\left(\lambda_{\text {up }}<\lambda_{\text {down }}\right)$.

A delta-doped layer has a finite thickness, in contrast to the Dirac delta function, which is a mathematical object. As shown in Fig. 1(b), the convolution of the DRF by a boxlike rectangular signal, featuring a constant atomic concentration and a finite thickness, is expected to describe a real and material delta-doping, and differs in its depth profile from the Dirac delta-function response. The SIMS response to a thin layer with sharp interfaces will appear wider, with smoother interfaces and a lower peak concentration. 
$1 \quad$ Isotope-enriched methane $\left({ }^{12} \mathrm{C}: 99.99 \%+{ }^{13} \mathrm{C}: 100 \mathrm{ppm}\right)$ was used to grow the buffer and the cap

2 diamond layers, while standard methane $\left({ }^{12} \mathrm{C}: 98.9 \%+{ }^{13} \mathrm{C}: 1.1 \%\right)$ was used to grow the delta layer in 3 diamond. In order to minimize surface roughness effects, a particularly flat sample was selected. The surface was ultra-polished in Japan by Syntek Co., Ltd.; the resulting surface RMS roughness, checked using a 3D optical profiler, was below $0.3 \mathrm{~nm}$ before overgrowth. In this study, $0.5 \mu \mathrm{m}$ of intrinsic buffer diamond was grown at the National Institute for Materials Sciences, NIMS (Tsukuba, Japan) in a high-plasma-density NIMS-type reactor with the ${ }^{12} \mathrm{C}$-enriched methane ${ }^{16)}$ in order to maintain a good crystalline quality and a flat surface. Then, a heavy boron-doped layer was grown at Institut Néel (Grenoble, France) in a modified NIRIM-type reactor designed for delta-doping diamond growth, from normal $\mathrm{CH}_{4}$ abundance $\left({ }^{12} \mathrm{C}: 98.9 \%\right)$ with $0.5 \%\left(\mathrm{CH}_{4} / \mathrm{H}_{2}\right)$ and $6000 \mathrm{ppm}(\mathrm{B} / \mathrm{C})_{\text {gas }}$ for 3 mins. ${ }^{11)}$ Please note that in the gas phase, the concentration of ${ }^{13} \mathrm{C}$ was only about twice that of boron. Finally, the top layer, i.e., cap layer, was grown at NIMS with isotopic-enriched methane to overgrow $50 \mathrm{~nm}$ of intrinsic diamond. The surface RMS roughness was close to $1 \mathrm{~nm}$ after the growth of the whole stack. No particular etching-back process including either oxygen or hydrogen was applied. ${ }^{5)}$ Thus, we report on a delta layer structure composed of isotopic carbon $\left({ }^{13} \mathrm{C}\right)$ and boron.

The synchronized boron and isotopic carbon diamond delta structure has been analyzed with a Cameca IMS 4f apparatus with $14.5 \mathrm{keV} \mathrm{Cs}^{+}$primary ions in $\mathrm{GEMaC}$, by collecting the negative secondary ions. ${ }^{13} \mathrm{C}$ and ${ }^{11} \mathrm{~B}$ depth profiles exhibit a similar behavior (Figs. 2 and 3 ), the boron incorporation following that of ${ }^{13} \mathrm{C}$ from methane, as expected. This observation illustrates the similarity in the fast kinetics of carbon and boron incorporation during growth.

The SIMS depth profile of ${ }^{13} \mathrm{C}$ intensity was fitted first (see Fig. 2) by the convolution of the depth resolution analytical function, eq. (1), and self-consistently with a rectangular function, already described in Fig. 1(b). The set of parameters used to create the DRF is shown in Table I, where $\delta$ represents the substituted structure thickness, i.e., the width of the rectangular function. 
These values were in good agreement with physical parameters. Indeed, the ion mixing simulated

2 by ion recoil of carbon atoms induced by the $\mathrm{Cs}^{+}$primary ion beam in a Monte Carlo method 3 algorithm $^{17)}$ was in the $3 \mathrm{~nm}$ range. This value was similar to the broad trailing edge $\left(\lambda_{\text {down }}\right)$. In addition,

4 the rising exponential component $\left(\lambda_{\text {up }}\right)$ of the DRF, which was around $1 \mathrm{~nm}$, was compatible with the

5 observed backscattering. The $50 \mathrm{~nm}$ thickness of the cap layer agreed with the position of the cusp $\left(z_{0}\right)$

6 in the double-exponential component of the DRF. The thickness of the delta layer measured using ${ }^{13} \mathrm{C}$

7 was compatible with the growth rate resulting from the homoepitaxy conditions, which was around 4.5

$8 \mathrm{~nm} \cdot \mathrm{min}^{-1}$. The difference between the rectangular profile and the ${ }^{13} \mathrm{C}$ SIMS depth profile as

9 deconvoluted using this DRF was of the same order as the noise level and justified the use of a box-like 10 rectangular function.

Fig. 3 shows the SIMS boron concentration profile $\left(\mathrm{P}_{\text {SIMS }}^{11 \mathrm{~B}}\right)$ along with the estimated position of the delta layer deduced from the fit of the ${ }^{13} \mathrm{C}$ DRF. This $\mathrm{P}_{\text {sIMs }}^{11 \mathrm{~B}}$ data was fitted by the convolution of the ${ }^{13} \mathrm{C}$ DRF and of a trapezoid function instead of a rectangular function, in order to take into account a possible mismatch. The nominal concentration of the estimated real dopant depth profile was determined from the integral under the SIMS boron concentration depth profile. For this operation, the parameters of the ${ }^{13} \mathrm{C}$ DRF were not modified. The reduction of the error between the fitting curve and the data was carried out by varying the trapezoid shape, i.e., by the optimization of rising and falling edge widths; the nominal concentration was supposed to be constant. Finally, the dopant boron concentration depth profile $\left(\mathrm{P}_{\text {Dopant }}^{11 \mathrm{~B}}\right)$ was deduced from the trapezoid function modulated by the Gaussian filtered noise resulting in the error fit and an offset of $2 \cdot 10^{17}$ at $\cdot \mathrm{cm}^{-3}$. Such a tentative deconvolution was made on finite elements, which depend on the spacing of the depth points recorded by SIMS. This procedure consequently introduces some errors, especially visible when the noise level is high. In this experiment, the SIMS energy was too high and the sputtering too fast to obtain a resolution good enough for precisely fitting the edge of the delta layer. However, after this DRF treatment, the boron 
1 profile was found to be 5 to 7 times sharper than before; the initially measured $1.5 \mathrm{~nm} / \mathrm{dec}$ rising edge

2 became $0.3 \mathrm{~nm} / \mathrm{dec}$ after fitting. This fitting gave the possibility of detecting a depth shift between the

3 concentration profiles of carbon and boron atoms (see Fig. 3). This could be coming from the late

4 boron incorporation (an $1.6 \mathrm{~nm}$-thick edge shift corresponds to a delay of $20 \mathrm{~s}$ for the gas mixture to

5 reach the nominal boron concentration on the diamond-growing surface) at the beginning of the boron-

6 delta layer growth, and from the residual boron contamination during the intrinsic cap-layer overgrowth.

7 In summary, we succeeded in extracting the depth resolution function (DRF) from a

8 synchronized $\mathrm{B}$ and ${ }^{13} \mathrm{C}$ diamond delta structure. By DRF deconvolution treatments, the broadening

9 induced by ion mixing has been reduced; the SIMS resolution has been improved and reached the

10 nanometer range. This allows a more reliable characterization by SIMS of nanometer-thin diamond-

11 embedded layers, containing specific doping (boron, nitrogen, or phosphorus), over many orders of

12 magnitude in concentration. For these reasons, the isotopic diamond delta structure is a powerful

13 calibration tool for SIMS.

Acknowledgments The financial support of Agence Nationale de la Recherche under contract ANR08-

BLAN-0195 and la Région Rhône-Alpes for the bourse de mobilité Explora'Doc are gratefully acknowledged. This work was also supported by the Strategic International Collaborative Research Project from the Japan Science and Technology Agency, and a Grant-in-Aid for Scientific Research from the Japan Society for the Promotion of Science (No. 18760241). The authors would like to express their deep gratitude to Professor G. Prudon for his kind expert advice on the depth resolution function treatment by isotope enrichment. 


\section{References}

1) H. Umezawa, M. Nagase, Y. Kato, and S. Shikata: Diamond Relat. Mater. 24 (2012) 201.

2) T. Ishikawa, K.-M. C. Fu, C. Santori, V. M. Acosta, R. G. Beausoleil, H. Watanabe, S. Shikata, and K. M. Itoh: Nano Lett. 12 (2012) 2083.

3) K. Ohno, F. J. Heremans, L. C. Bassett, B. A. Myers, D. M. Toyli, A. C. Bleszynski Jayich, C. J. Palmstrøm, and D. D. Awschalom: Appl. Phys. Lett. 101 (2012) 082413.

4) R. Edgington, S. Sato, Y. Ishiyama, R. Morris, R. B. Jackman, and H. Kawarada: J. Appl. Phys. 111 (2012) 033710 .

5) A. Fiori, T. N. Tran Thi, G. Chicot, F. Jomard, F. Omnès, E. Gheeraert, and E. Bustarret: Diamond Relat. Mater. 24 (2012) 175.

6) S. Hofmann: App. Phys. 13 (1977) 205.

7) R. S. Balmer, I. Friel, S. Heppelstone, J. Isberg, M. J. Uren, M. L. Markham, N. L. Palmer, J. Pilkington, P. Huggett, S. Majdi, and R. Lang: J. Appl. Phys. 113 (2013) 033702.

8) Y. Shimizu, H. Takamizawa, Y. Kawamura, M. Uematsu, T. Toyama, K. Inoue, E. E. Haller, K. M. Itoh, and Y. Nagai: J. Appl. Phys. 113 (2013) 026101.

9) D. Araujo, P. Achatz, R. El Bouayadi, A. J. Garcia, M. P. Alegre, M. P. Villar, F. Jomard, and E. Bustarret: Diamond Relat. Mater. 19 (2010) 972.

10) Y.-G. Lu, S. Turner, J. Verbeeck, S. D. Janssens, P. Wagner, K. Haenen, and G. Van Tendeloo: Appl. Phys. Lett. 101 (2012) 041907.

11) G. Chicot, T. N. Thu Nhi, A. Fiori, F. Jomard, E. Gheeraert, E. Bustarret, and J. Pernot: Appl. Phys Lett. 101 (2012) 162101.

12) Y. Shimizu, A. Takano, and K. M. Itoh: Appl. Surf. Sci. 255 (2008) 1345.

13) H. Watanabe, and S. Shikata: Diamond Relat. Mater. 20 (2011) 980.

14) M. G. Dowsett, G. Rowlands, P. N. Allen, and D. Barlow: Surf. Interface Anal. 21 (1994) 310.

15) B. Gautier, R. Prost, G. Prudon, and J. C. Dupuy: Surf. Interface Anal. 24 (1996) 733.

16) T. Teraji, T. Taniguchi, S. Koizumi, K. Watanabe, M. Liao, Y. Koide, and J. Isoya: Jpn. J. Appl. Phys. 51 (2012) 090104.

17) J. F. Ziegler: SRIM 1984-2013. 


\section{Caption Fig. 1 .}

Simulations of SIMS depth profiles corresponding to (a) a Dirac delta function (pink vertical line localized at $47 \mathrm{~nm}$ below the surface) with the corresponding DRF (yellow filled black line) and (b) a 14 nm-thick layer (pink filled box) with the corresponding DRF convolution (construction elements symbolized by the yellow pattern). On such profiles, the surface lies on the left at "0" depth.

\section{Caption Fig. 2.}

Depth profiles for ${ }^{13} \mathrm{C}$ concentrations in the diamond delta structure, plotted in linear (left) and semilog (right) scales. The SIMS intensity depth profile $\left(\mathrm{P}_{\text {sims }}^{13 \mathrm{C}}\right)$ is represented by purple dots; the dashed black line follows the corresponding fit by the convolution of the DRF, and square input signal. The DRF is plotted as a solid blue line and the ${ }^{13} \mathrm{C}$ dopant profile $\left(\mathrm{P}_{\mathrm{Dopant}}^{13 \mathrm{C}}\right)$ corresponding to the deconvolution of $\mathrm{P}_{\mathrm{sims}}^{13 \mathrm{C}}$ by the DRF is plotted in orange.

\section{Caption Fig. 3.}

Depth profiles related to ${ }^{11} \mathrm{~B}$ in the diamond delta structure plotted in linear (left) and semilog scales. Light green and black dots follow the SIMS boron concentration profile $\left(\mathrm{P}_{\text {sims }}^{11 \mathrm{~B}}\right)$ and the deconvoluted boron dopant profile $\left(\mathrm{P}_{\mathrm{Dopant}}^{11 \mathrm{~B}}\right)$ respectively. The orange box indicates the position of the delta layer based on the ${ }^{13} \mathrm{C}$ dopant profile. 
Table I. Parameters of the DRF used in the fitting process. $\mathrm{Cs}^{+}$primary ions were used at $14.5 \mathrm{keV}$, and the incidence angle was $27^{\circ}$ in a Cameca IMS $4 \mathrm{f}$.

\begin{tabular}{ccccc}
\hline $\begin{array}{c}\lambda_{\text {up }} \\
(\mathrm{nm})\end{array}$ & $\begin{array}{c}\lambda_{\text {down }} \\
(\mathrm{nm})\end{array}$ & $\begin{array}{c}\sigma \\
(\mathrm{nm})\end{array}$ & $\begin{array}{c}z_{0} \\
(\mathrm{~nm})\end{array}$ & $\begin{array}{c}\delta \\
(\mathrm{nm})\end{array}$ \\
\hline 0.9 & 3.0 & 0.6 & 47 & 14 \\
\hline
\end{tabular}


Fig. 1.

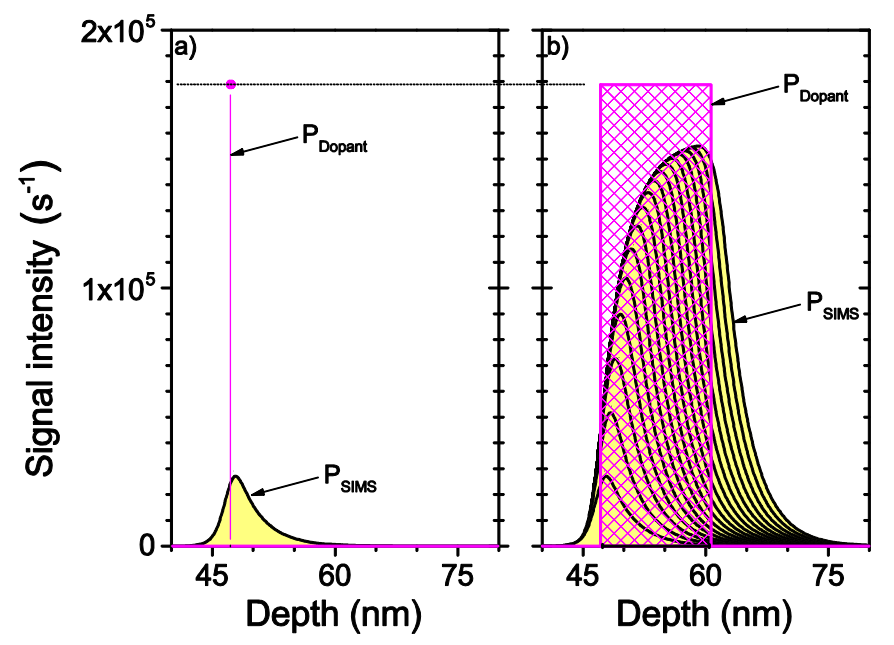

Fig. 2.

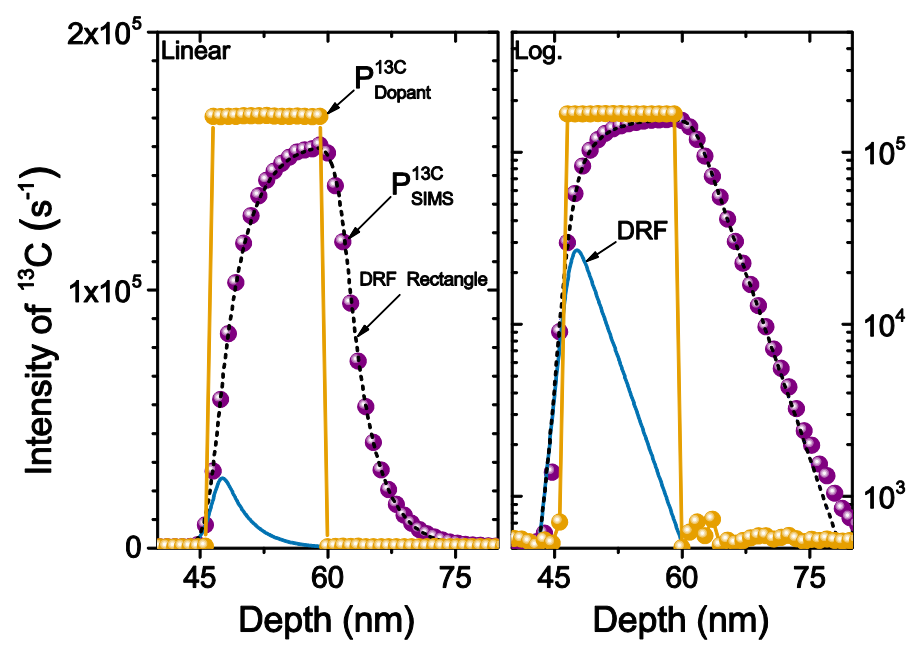

Fig. 3.

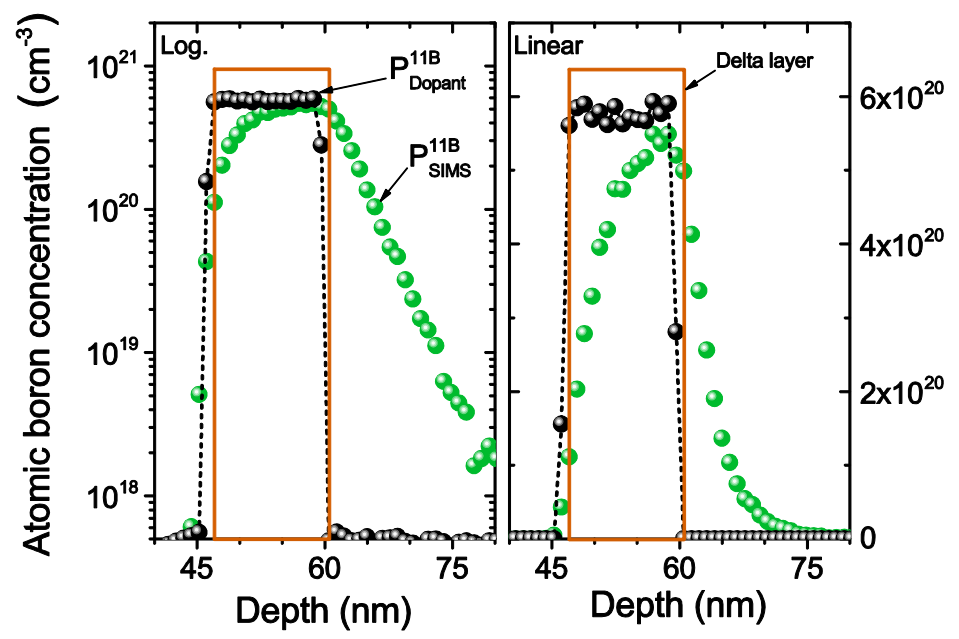

